\title{
DISTRIBUIÇÃO DE Ca, Ba, Sr, E Rb ENTRE LEUCITA E "MAGMA" SANIDÍNICO A 2 kbar E $1030^{\circ} \mathrm{C}$
}

\author{
ANA HELENA PACHECO MOREIRA*, JOSÉ VICENTE VALARELLI** e FRANÇOIS DELBOVE***
}

\begin{abstract}
DISTRIBUTION OF $\mathrm{Ca}, \mathrm{Ba}, \mathrm{Sr}$, AND Rb BETWEEN LEUCITE AND SANIDINIC MAGMA AT $2 \mathrm{kbar}$ AND $1030^{\circ} \mathrm{C}$. Data from experimental works concerning the distribution coefficients of $\mathrm{Rb}, \mathrm{Ca}, \mathrm{Sr}$ and Ba between leucite and hidrothennal solutions and between silico-sanidinic fusion and equivalent solutions (at $1.030^{\circ} \mathrm{C}$ and $2 \mathrm{kbar}$ ) were used to obtain the distribution coefficients between leucite and experimental sanidinic magma. These data are: $\mathrm{Kd}_{\mathrm{Rb}}=2.36 ; \mathrm{Kdc}_{\mathrm{a}}=0.088 ; \mathrm{Kd}_{\mathrm{Sr}}=$ 0.12 ; and; $\mathrm{Kd}_{\mathrm{Ba}}=1.89$. They show that the $\mathrm{Rb}$ and $\mathrm{me} \mathrm{Ba}$ wouldprefer better the leucite than the experimental "magmatic liquid and that $\mathrm{Ca}$ and the $\mathrm{Sr}$ have opposite behavior. Normalized calculations based on these data and on thermodynamic considerations about the reactions permit the contraction of graphical variation of the relations trace elements $/ \mathrm{K}$ and trace element $\mathrm{I} /$ trace element 2 as function of degree of leucite crystallization. As well the leucite grows in equilibrium from the insaturated (system $\mathrm{KAlSi}_{2} \mathrm{O}_{6}-\mathrm{SiO}_{2}$ ), experimental "magma", the ratios $\mathrm{Ca} / \mathrm{K}$ and $\mathrm{Sr} / \mathrm{K}$ increase exponentially and the ratios $\mathrm{Ba} / \mathrm{K}$ and $\mathrm{Rb} / \mathrm{K}$ decrease gently on the residual experimental magmatic liquid. At the same time, the ratio $\mathrm{Rb} / \mathrm{Ba}$ decreases slowly and the ratios $\mathrm{Ba} / \mathrm{Sr}$ and $\mathrm{Rb} / \mathrm{Sr}$ decrease considerably, under the experimental conditions.
\end{abstract}

Keywords: Thermodynamic, leucite, sanidinic magma.

\begin{abstract}
RESUMO Baseando-se em dados experimentais de coeficientes de partição de $\mathrm{Rb}, \mathrm{Ca}$, Sr e Ba entre leucita e soluções hidrotermais e entre fusões sílico-sanidínicas e soluções hidrotermais equivalentes, a $1.030^{\circ} \mathrm{C}$ e 2 kbar, são estabelecidos os coeficientes de partição desses oligoelementos entre a leucita e magma sanidínico, nas mesmas condições de PT. Esses valores são $\mathrm{Kd}_{\mathrm{Rb}}=2,36 ; \mathrm{Kd}_{\mathrm{Ca}}=0,088 ; \mathrm{Kd}_{\mathrm{Sr}}=0,12 \mathrm{e}$ $\mathrm{Kd}_{\mathrm{Ba}}=1,89$; e demonstram a preferencia do $\mathrm{Rb}$ e do Ba pela leuata e do Ca e $\mathrm{Sr}$ pela fusão sílico-sanidínica. Com ajuda desses coeficientes, das reações de troca iônica entre as fases envolvidas e de equações de equilíbrio, são estabelecidos gráficos normalizados da variação das relações elemento traço/potássio e elementos traço entre si, em função da cristalização de leucita em equilíbrio com "magma" sílico-sanidínico experimental. Com o avanço da cristalização da leucita, em condições de equilíbrio, a partir de um "magma" alcalino insaturado do sistema $\mathrm{KAlSi}_{2} \mathrm{O}_{6}-\mathrm{SiO}_{2}$, as relações $\mathrm{Ca} / \mathrm{K}$ e Sr$/ \mathrm{K}$ aumentam exponencialmente no "magma residual", ao passo que $\mathrm{Ba} / \mathrm{K}$ e $\mathrm{Rb} / \mathrm{K}$ diminuem ligeiramente nesse mesmo "magma". Nessas mesmas condições, a relação $\mathrm{Rb} / \mathrm{Ba}$ diminui ligeiramente quando comparada com a mesma razão do "magma" inicial, e as relações $\mathrm{Ba} / \mathrm{Sr}$ e $\mathrm{Rb} / \mathrm{Sr}$ diminuem consideravelmente dentro das condições experimentais.
\end{abstract}

Palavras-chaves: Termodinâmica, leucita, magma sanidfnico.

INTRODUÇÃO Por meio de experiências de síntese hidrotermal a $1.030^{\circ} \mathrm{C}$ e 2 kbar, Moreira et al. (1989a,b,c,d) determinaram os coeficientes de distribuição de $\mathrm{Ca}, \mathrm{Ba}, \mathrm{Sr}$ e $\mathrm{Rb}$ entre leucita (KA1Sí $\mathrm{O}_{6}$ ) e soluções hidrotermais $(\mathrm{SH})$, e entre fusões sílico-sanidínicas $\left(0,93 \mathrm{KAlSi}_{3} \mathrm{O}_{6}-0,07 \mathrm{SiO}_{2}\right)$ e soluções hidrotermais equivalentes. A escolha da composição da fusão sílico-sanidínica é explicada pela composição do ponto peritético do sistema Lc-Qz (Shaw 1963) (Fig. 1).

Os materiais de partida para a síntese dos sólidos eram constituídos por mistura de gels amorfos na estequiometria desejada; e as soluções de partida eram constituídas por solução aquosa de $\mathrm{KC1}$ e cloretos de $\mathrm{Ca}, \mathrm{Ba}, \mathrm{Sr}$ e $\mathrm{Rb}$, nas quais teores conhecidos de elementos radioativos ${ }^{133} \mathrm{Ba},{ }^{85} \mathrm{Sr},{ }^{86} \mathrm{Rb}$ e ${ }^{45} \mathrm{Ca}$ facilitaram as análises desses elementos em traços sólidos (cristais de leucita ou vidro sílico-sanidínico) e nas soluções.

Nesses trabalhos foram estabelecidos os coeficientes de distribuição dos oligoelementos e os limites de concentrações até os quais as distribuições são lineares, refletindo comportamento ideal, segundo a lei de Henry, das fases sólidas (extremamente diluídas) e das soluções nidrotermais. A tabela 1 resume esse resultados.

DISTRIBUIÇÃO DOS ELEMENTOS Distribuição do Rubídio A reação de troca iônica $\mathrm{Rb}-\mathrm{K}$ entre a leucita sintetizada e a solução hidrotermal por ser expressa por:

$\left(\mathrm{KAlSi}_{2} \mathrm{O}_{6} h_{\circ \mathrm{C}}+(\mathrm{RbCl})_{\mathrm{SH}}=\left(\mathrm{RbAlSi}_{2} \mathrm{O}_{6} h_{\alpha C}+(\mathrm{KCI})_{\mathrm{SH}}\right.\right.$
Tabela l - Coeficiente de partição de Rb, Ca, Sr e Ba entre leucita cristalizada ( $\mathrm{LcC}$ ) e solução hidrotermal (SH) e entre sarúdina fundida (SnF) e SH obtidos por meio de experimentos de sintese a $1.030^{\circ} \mathrm{C}$ e $2 \mathrm{kbar}$, acompanhados dos limites de ideatidade (Henry) e expressos em ppm do elemento traço nos "soados" (Moreira et al. 7959a, b, c, d)

Table 1 - Distribution coefficients of $\mathrm{Rb}, \mathrm{Ca}, \mathrm{Sr}$ and $\mathrm{Ba}$ between crystallized leucite (LcQ and hydrothermal solution $(\mathrm{SH})$ and between fused sanidine $(\mathrm{SnF})$ and $\mathrm{SH}$ obtained by means of synthesis experiments at $1.030^{\circ} \mathrm{C}$ and $2 \mathrm{kbar}$, with ideality limits (Henry) and expressed in ppm of trace element in the "solids" (Moreira et al. 1989a, b, c, d)

\begin{tabular}{|c|c|c|c|c|c|c|c|c|}
\hline \multirow[b]{2}{*}{$\begin{array}{l}\text { Cosilelents: } \\
\text { de Partiotio }\end{array}$} & \multicolumn{4}{|c|}{ Elomentos } & \multicolumn{4}{|c|}{ 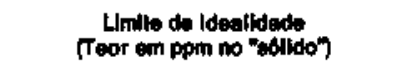 } \\
\hline & Fio & G* & $\mathbf{3 r}$ & Ba & fit & Ga & $9 r$ & $\mathrm{Ba}$ \\
\hline Kd $1.000^{\circ} \mathrm{C}$ & 1,30 & 0,69 & 1,06 & 17,00 & $t 0,200$ & 2,000 & 200 & 9400 \\
\hline $\mathrm{Kd} \mathrm{SnF}_{\mathrm{SnH}}^{1.000^{\circ} \mathrm{C}}$ & 0,55 & 6,70 & 8,50 & 9.0 & $>150$ & $>500$ & $>10,000$ & $>80.000$ \\
\hline
\end{tabular}

A reação de troca $\mathrm{Rb}-\mathrm{K}$ entre a fusão sanidínica sintetizada e a solução hidrotermal pode ser representada por:

$\left(\mathrm{KAlSi}_{3} 0_{8}\right)_{\mathrm{SnF}}+(\mathrm{RbCl})_{\mathrm{S}} \mathrm{H}=\left(\mathrm{RbAlSi}_{3} \mathrm{O}_{8}\right)_{\mathrm{SnF}}+(\mathrm{KC} 1)_{\mathrm{SH}}(2)$ onde $\mathrm{LcC}=$ leucita cristalina, $\mathrm{SH}=$ solução hidrotermal e $\mathrm{SnF}=$ sanidina fundida nas condições experimentais ou vidro sanidínico nas condições ambientes.

\footnotetext{
* Instituto de Química, Universidade Federal Fluminense, Outeiro de São Joio Batista, s/n, Centro, CEP 24000, Niterói, RJ, Brasil

** Departamento de Mineralogia e Petrologia, Instituto de Geociências, Universidade de São Paulo, Caixa Postal 20899, CEP 01498, São Paulo, SP, Brasil

*** Centre de Recherches sur Ia Synthèse et Chimie dês Minéraux, CNRS IA, Rue de La Ferollerie 45075, Orleans, Cedex, France
} 


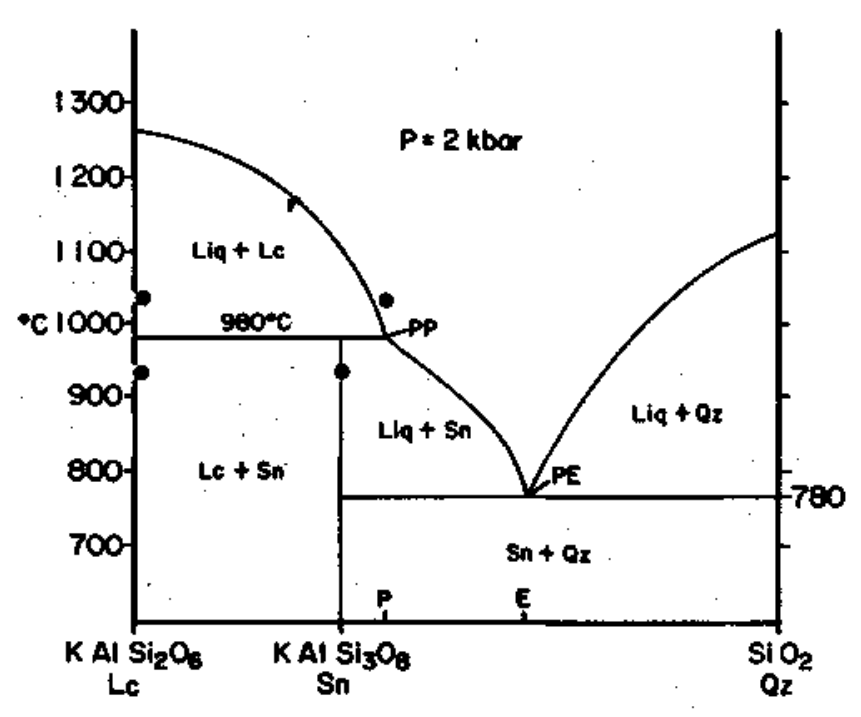

Figura l - Diagrama de fase do sistema $\mathrm{KAlSi}_{2} \mathrm{O}_{6}-\mathrm{SiO}_{2}$ a 2 kbar, adaptado de Show (1963), mostrando as condições de temperatura e de composição mássica dos experimentos (Moreira 1988). $P P=$ ponto peritético; $P E=$ ponto eutético; $P=$ composição pernética e E = composição eutética; $\mathrm{Liq}=$ líquido; Lc = leucüa;Sn = sanidina; $Q z=$ quartzo Figure 1 - Phase diagram for $\mathrm{KAlSi}_{2} \mathrm{O}_{6}-\mathrm{SiO}_{2}$ system at $2 \mathrm{kbar}$, adapted from Shaw (1963), showing the conditions of temperature and mass composition of experiments (Moreira 1988). $\mathrm{PP}=$ peritetic point; $\mathrm{PE}=$ eutectic point; $\mathrm{P}=$ peritetic composition; $\mathrm{Liq}=$ liquid; $\mathrm{Lc}=$ leucite; $\mathrm{Sn}=$ sanidine; $\mathrm{Qz}=$ quartz

Com todo o rigor, a reação (2) deveria ser escrita como a troca $\mathrm{Rb}-\mathrm{K}$ entre fusão sílico-sanidínica e $\mathrm{SH}$ :

\section{$1,075\left(0,93 \mathrm{KAISt}_{3} \mathrm{O}_{6} 0,07 . \mathrm{SiO}_{2}\right)_{\mathrm{sm}}+(\mathrm{RbCl})_{\mathrm{SH}}=$$$
=1,075\left(0,93 \mathrm{RbAlSi}_{3} \mathrm{O}_{8} 0,07 . \mathrm{SiO}_{2}\right)_{\mathrm{Snf}}+(\mathrm{KCD})_{\mathrm{SH}}
$$

No entanto, o pequeno excesso de sílica nas suas fusões, potássica ou rubídica, e dado os teores muito baixos deste último elemento, à relação (2) simplificada pode ser usada.

As equações de equílibrio das duas reações podem ser expressas por:

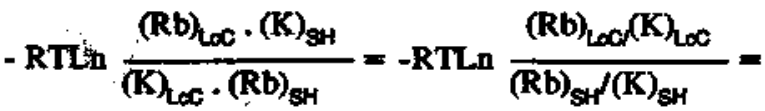

$$
\begin{aligned}
& =-\operatorname{RTLnKd}_{\mathrm{Ab}, \mathrm{LO}-\mathrm{gH}}=(\Delta G)_{\mathrm{RD}, \mathrm{LO}-\mathrm{SH}}
\end{aligned}
$$$$
\text { - RTLn } \frac{(\mathrm{Rb})_{\mathrm{Sn}} \cdot(\mathrm{K})_{\mathrm{SH}}}{(\mathrm{K})_{\mathrm{SN}} \cdot(\mathrm{Rb})_{\mathrm{SH}}}=-R T L n \frac{(\mathrm{Rb})_{\mathrm{Snf}} /(\mathrm{K})_{\mathrm{SnF}}}{(\mathrm{Rb})_{\mathrm{gH}} /(\mathrm{K})_{\mathrm{gH}}}=
$$$$
=-\operatorname{RTLnKd}_{\mathrm{Pb}, \mathrm{SnF}, \mathrm{SH}}=(\Delta G)_{\mathrm{Rb}, \mathrm{SnF}-\mathrm{SH}}
$$

Essas equações são válidas para os limites de linearidade dos valores dos coeficientes de distribuição $(\mathrm{Kd})$ determinados experimentalmente, que eqüivale a considerar as soluções sólidas e as soluções hidrotermais como ideais, segundo a lei de Henry $(\mathrm{T}=$ Temperatura em graus Kelvin). As quantidades $(\mathrm{Rb})$ e $(\mathrm{K})$ representam as frações molares do $\mathrm{Rb}$ e do $\mathrm{K}$ nas fases consideradas:
$(\mathbf{R b})_{\mathrm{S} \cap F}=\frac{\mathbf{R b}}{\mathbf{R b}+\mathbf{K}}$ na samidina fundida;
$(\mathbf{R b})_{S H}=\frac{\mathbf{R b}}{\mathbf{R} \mathbf{b}+\mathrm{K}}$, da soluça hidrotermal.

$(\Delta G)_{\mathrm{Fb}, L C C-S H}$ e $(\Delta G)_{\mathrm{Hb}, S n F-s H}$ sho, respectivamente, as energias livres de Gibbs das reaçes de troca (1) e (2).

Combinando-se as reaçóes (1) e (2), obtem-se:

$\left(\mathrm{KAlSi}_{2} \mathrm{O}_{6} h_{\mathrm{OC}}+\mathrm{RbAISi}_{3} \mathrm{O}_{8}\right)_{\mathrm{SnF}}=$

$$
=\left(\mathrm{RbAlSi}_{2} \mathrm{O}_{6}\right)_{\mathrm{LC}}+\left(\mathrm{KAISi}_{3} \mathrm{O}_{6}\right)_{9 \mathrm{FF}}
$$

que representa a troca iônica $\mathrm{Rb}-\mathrm{K}$ entre leucita cristalizada e magma sanidínico a $1030^{\circ} \mathrm{C}$ e 2 kbar ou partição dos dois elementos entre leucita em equílibrio com a fusão, cuja equação de equilíbrio é:

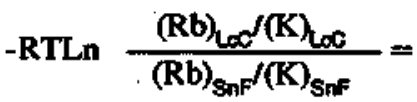

$$
\begin{aligned}
& =-R T L n K d_{\mathrm{Rb}, L O C-S \mathrm{nF}}=(\Delta G)_{\mathrm{Rb}, L \mathrm{LC}-\$ \mathrm{NF}}
\end{aligned}
$$

que por sua vez, reaçio (1) - (2), pode ser escrita:

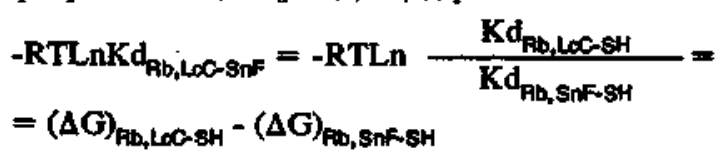

Conhecendo-se os valores de $\mathrm{Kd}$ entre as fases $\mathrm{LcC}$ e $\mathrm{SnF}$ e as soluções hidrotermais, é possível estabelecer o coeficiente de partição do $\mathrm{Rb}$ entre a leucita cristalizada e a fusão sanidínica $=1,30 / 0,55=2,36$ (Tabelas 1 e 2$)$.

Outras considerações termodinâmicas podem ser vistas em Moreira (1988), Delbove (1978) e Lagache (1974a e b).

Distribuição dos elementos alcalinos terrosos As reações que exprimem a troca do potássio da leucita ou do magma sanidínico por um elemento alcalino terroso, de $\mathrm{SH}$, a exemplo do cálcio, são as seguintes:

$$
\begin{aligned}
& 2\left(\mathrm{KAISi}_{2} \mathrm{O}_{6}\right)_{\infty \mathrm{C}}+\left(\mathrm{CaCl}_{2}\right)_{\mathrm{SH}}= \\
& =\left[\mathrm{CaO}\left(\mathrm{AlSi}_{2} \mathrm{O}_{6}\right)_{2 \infty}+2(\mathrm{KCl})_{\mathrm{SH}}\right.
\end{aligned}
$$

ou

$$
\begin{aligned}
& 2\left(\mathrm{KAISi}_{2} \mathrm{O}_{9} \lambda_{\infty \mathrm{C}}+(\mathrm{CaCl})_{\mathrm{SH}}=\right. \\
& =\left(\mathrm{CaAl}_{2} \mathrm{SiO}_{6} h_{\infty}+2(\mathrm{KCl})_{S H}+3\left(\mathrm{SiO}_{2}\right)\right.
\end{aligned}
$$

$$
\begin{aligned}
& 2\left(\mathrm{KAISi}_{9} \mathrm{O}_{9}\right)_{\text {Sh }}+\left(\mathrm{CaCl}_{2}\right)_{\text {SH }}= \\
& =\left[\mathrm{Ca}\left(\mathrm{Al}_{2} \mathrm{Si}_{6} \mathrm{O}_{10}\right)\right]_{\mathrm{SnF}}+2(\mathrm{KCl})_{\mathrm{SH}}
\end{aligned}
$$

ou

$$
\begin{aligned}
& 2\left(\mathrm{KAISt}_{\mathrm{g}} \mathrm{O}_{\mathrm{g}}\right)_{\mathrm{anF}}+(\mathrm{CaCl})_{\mathrm{hh}}= \\
& =\left(\mathrm{CaAl}_{2} \mathrm{Si}_{2} \mathrm{O}_{2}\right)_{\mathrm{SF}}+2\left(\mathrm{KCl}_{\mathrm{gH}}+4\left(\mathrm{SiO}_{2}\right)\right.
\end{aligned}
$$

en que $\mathrm{D}=$ vactencia reticular.

$\mathrm{Na}$ análise por difração de raios $\mathrm{X}$ não foi constatada a presença de quartzo como nos trabalhos experimentais de Wyart \& Sabatier (1965) e liyama (1972). Acrescente-se o 
fato de que, nas condições experimentais, o quartzo é incompatível. Se porventura a reação $(9 b)$ predominasse sobre a (9a), a sílica somente poderia estar dissolvida na solução hidrotermal. $\mathrm{Na}$ fusão, a sílica ficará dissolvida no magma sílico-sanidfnico, conservando-se sempre a relação $\mathrm{Si} / \mathrm{Al}$ do sistema. O grau de substituição do $\mathrm{Si}$, nas condições experimentais, pode ser desprezado em vista da diluição dos elementos traços alcalinos terrosos.

As respectivas equações de equilíbrio são:

$-\operatorname{RTLn} \frac{(\mathrm{Ca})_{\mathrm{Lod}} /(\mathrm{K})_{\mathrm{LOC}}^{2}}{(\mathrm{Ca})_{\mathrm{SH}} /(\mathrm{K})_{\mathrm{SH}}^{2}}=-\mathrm{RTL} \dot{\mathrm{nK}} \mathrm{Cd}_{\mathrm{Ca}, \mathrm{LO}-\mathrm{SH}}=$

$(\Delta \mathrm{G})_{\mathrm{Cu}, \mathrm{LOC-SH}}$

ou

$\left.-\operatorname{RTLn} \frac{(\mathrm{Ca})_{\mathrm{LOO}} /(\mathrm{K})^{2}{ }_{\mathrm{LOC}}}{(\mathrm{Ca})_{\mathrm{SH}} /(\mathrm{K})^{2}{ }_{\mathrm{SH}}}{ }_{\mathrm{SH}_{\mathrm{a}}}\right)^{3}=$

$=-\operatorname{RTLnKd}_{\mathrm{Ca}, L O C-S H}+3 \operatorname{RTLn}\left(\mathrm{a}_{\mathrm{shO}}\right)=$

$(\Delta \mathrm{G})_{\mathrm{Ca}_{1, L C S H H}}+3 \mathrm{G}^{\mathrm{SiO}}$

$-\operatorname{RTLn} \frac{(\mathrm{Ca})_{\mathrm{SnF}} /(\mathbf{K})^{2}{ }_{\mathrm{SnF}}}{(\mathrm{Ca})_{\mathrm{SH}} /(\mathrm{K})^{2}{ }_{\mathrm{SH}}}=$

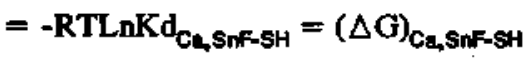

(12a)

ou

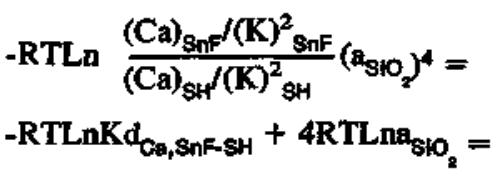

$(\Delta \mathrm{G})_{\mathrm{Co}, \mathrm{BnF}-\mathrm{gH}}+4 \mathrm{G}^{\mathrm{SNO}}$

Considerando-se as reaçóes (9a) e (10a) como representantes da troca iônica Ca-K entre leucita cristalizada e magna sanidínico à $1030^{\circ}$ e 2 kbar (9a)-(10a), tem-se:

$2\left(\mathrm{KAlSi}_{2} \mathrm{O}_{6}\right)_{\mathrm{Lac}}+\left[\mathrm{Ca}\left(\mathrm{Al}_{2} \mathrm{Si}_{6} \mathrm{O}_{16}\right)\right]_{\mathrm{SnF}_{\mathrm{F}}}=$

$=\left[\mathrm{CaO}\left(\mathrm{AlSi}_{2} \mathrm{O}_{6}\right)_{2} \mathrm{lLOC}_{\mathrm{LC}}+2\left(\mathrm{KAlSi}_{8} \mathrm{O}_{8}\right)_{\mathrm{SnF}}\right.$

com

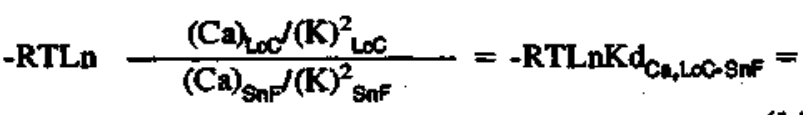

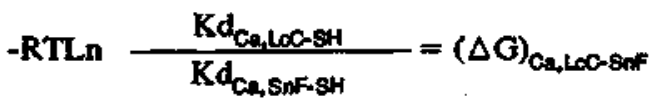

Considerando-se as reagoes (9b) e (10b), esse equfibrio e dado por:

$$
\begin{aligned}
& 2\left(\mathrm{KAlSi}_{2} \mathrm{O}_{4}\right)_{\mathrm{LxC}}+\left(\mathrm{CaAl}_{2} \mathrm{Si}_{2} \mathrm{O}_{6}\right)_{\text {SnF }}+\mathrm{SiO}_{2}= \\
& 2\left(\mathrm{KAISi}_{3} \mathrm{O}_{6}\right)_{\mathrm{SnF}}+\left(\mathrm{CaAl}_{2} \mathrm{Si}_{2} \mathrm{O}_{3}\right)_{\mathrm{LC}}
\end{aligned}
$$

As reações (13a) e (13b) são muito semelhantes, com excessão da sílica da reação (13b), cuja quantidade é .mínima, uma vez que ela é dependente do teor de cálcio que se encontra como traço.

Dessa forma, as relações entre os coeficientes de partição estabelecidos na equação de equílibrio (14a) seriam válidas para a reação (13b). Não é o mesmo em relação a $(\Delta \mathrm{G})$.

Os coeficientes de partição dos elementos alcalinos terrosos como traços, dentro dos limites de idealidade (Henry), entre leucita e magma sanidínico, são fornecidos na tabela 2.

Tabela 2 - Coeficiente indiretos de distribuição de $\mathrm{Ca}, \mathrm{Sr}, \mathrm{Ba}$ $e$ Rb entre leucita e fusão sílico-sanidínica ("magma" experimental) a $1.030^{\circ} \mathrm{C}$ e $2 \mathrm{kbar}$

Table 2 - Indirect distribution coefficient of $\mathrm{Ca}, \mathrm{Sr}, \mathrm{Ba}$ and $\mathrm{Rb}$ between leucite and silicic - sanidinic fusion (experimental "magma" at $1.030^{\circ} \mathrm{C}$ and $2 \mathrm{kbar}$

\begin{tabular}{|c|c|c|c|}
\hline & ${ }^{\mathrm{Kd}}{ }_{\text {LoC-SH }}$ & $\mathrm{Kd}_{\text {SnF-SH }}$ & $\mathrm{Kd}_{\text {LeC-SnF }}$ \\
\hline Ca & 0,59 & 6,70 & 0,088 \\
\hline Sr & 1,05 & 8,50 & 0,12 \\
\hline Ba & 17,00 & 9,00 & 1,89 \\
\hline
\end{tabular}

Outras considerações termodinâmicas a respeito dos equilíbrios das reações em questão podem ser vistas em Moreira (1988), Delbove (1978) é Volfinger \& Robert (1980).

COMPORTAMENTO DE Rb, Ca, Sr E Ba DURANTE A CRISTALIZAÇÃO DA LEUCITA O diagrama da figura 1 estabelece o campo de estabilidade das fases do sistema $\mathrm{KAlSi}_{2} \mathrm{O}_{6}-\mathrm{SiO}_{2}$, em função da composição e da temperatura, à pressão total constante igual à pressão de fluídos, $2 \mathrm{kbar}$. Nessa mesma figura acham-se representados os pontos das sínteses de leucita e das fusões sílico-sanidínicas cujos equilíbrios foram estabelecidos pelas reações (1) e (2) para o $\mathrm{Rb}$ e pelas reações (9) e (10) para os demais alcalinos terrosos. Como conseqüência desses experimentos, foram estabelecidas as reações (6) para o $\mathrm{Rb}$ e (13) para o $\mathrm{Ca}$.

Esses equilíbrios envolvem a fase cristalina leucita, o líquido magmático de composição peritétíca, à temperatura um pouco acima $\left(50^{\circ} \mathrm{C}\right)$ da temperatura peritétíca e a solução hidrotermal. Qualquer que seja a composição de um magma inicial insaturado em sílica (do lado esquerdo da composição peritétíca), a leucita se critaliza a partir desse magma por resfriamento e o magma residual vai se enriquecendo em sílica, tendendo a se aproximar da composição da fusão sílico-sanidínica dos experimentos.

As reações mencionadas acima, válidas nas condições experimentais, provavelmente podem ser extrapoladas em até $100^{\circ} \mathrm{C}: 50^{\circ} \mathrm{C}$ acima das condições experimentais e $50^{\circ} \mathrm{C}$ abaixo até a temperatura peritética. Além disso, pode-se dizer que elas são válidas para as leucitas cristalizadas a temperaturas maiores (magmas muito pobres em sílica), desde que as trocas tônicas entre cristal já formado e magma atinjam o equilíbrio no intervalo de $100^{\circ} \mathrm{C}$ de temperatura antes de atingir Tp. Essa condição seria realizada em resfriamento lento.

Imaginando-se uma certa massa de magma do sistema considerado, a $2 \mathrm{kbar}$, do qual se cristaliza leucita; que esse magma seja saturado em água, com $\mathrm{KC} 1$, contendo uma quantidade de um elemento traço cuja concentração seja compatível com as dos experimentos; que o magma constitui uma fase única da qual começa a se cristalizar leucita, em condições de 
equilíbrio perfeito com o líquido magmático residual e com uma solução hidrotermal cujo título seja semelhante ao dos experimentos $\sim 1.5 \mathrm{~N}$; é possível calcular a distribuição do potássio e de um elemento traço considerado entre a leucita cristalizada, o líquido residual e a solução hidrotermal em função do grau de cristalização (\% de cristalização) da leucita. Esse cálculo é baseado nos coeficientes de distribuição das tabelas 1 e 2 . As figuras 2 a 3 mostram os resultados desses cálculos, respectivamente, para $\mathrm{Rb}$ e $\mathrm{Ba}$ e para $\mathrm{Ca}$ e $\mathrm{Sr}$.

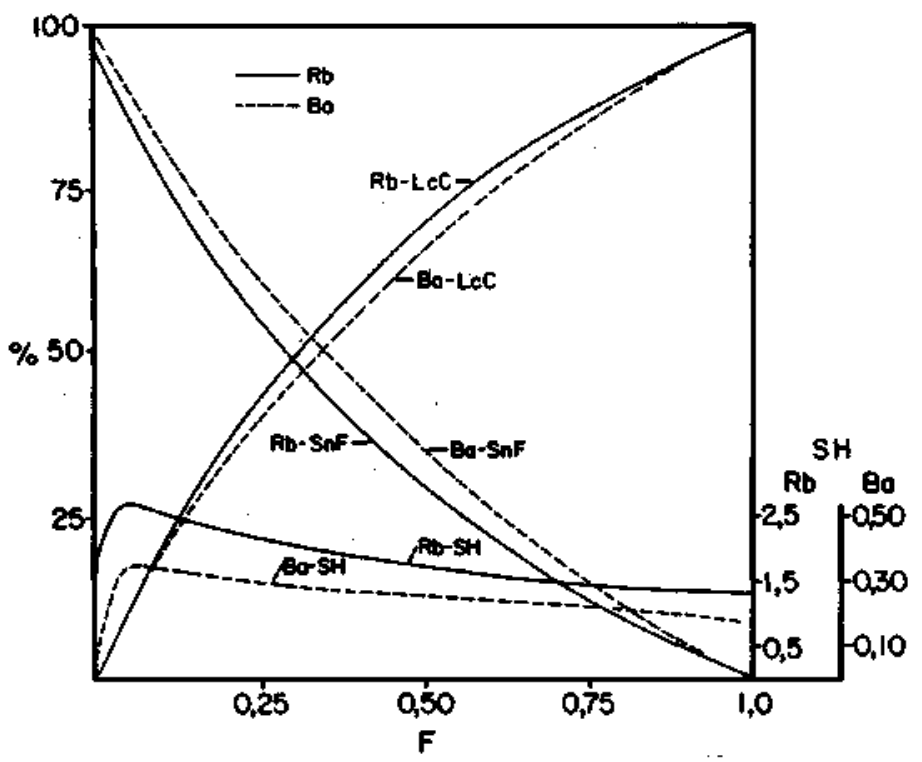

Figura 2 - Evolução da distribuição de Rb e Ba entre leucita cristalizada ( $L c C$ ), solução hidrotermal (SH) e fusão sttico-sanidínica (SnF) em função de seus teores no magma primitivo, homogêneo e saturado ( 10\%) em água, à medida que a leucita se cristaliza. A escala das porcentagens desses elementos na solução hidrotermal foi sobrelevada para maior clareza. F = grau de cristalização da leucita

Figure 2 - Evolution of $\mathrm{Rb}$ and $\mathrm{Ba}$ distributed among crystalized leucite (LcC), hydrothermal solution ( $\mathrm{SH}$ ) and silic-sanidinic fusion as function of their concentrations in original magma, homogeneous and water saturated $(\sim 10 \%)$, in order of crystallization of leucite. The scale of these elements in hydrothermal solution was magnified. $\mathrm{F}=$ degree of crystallization of leucite

Como conseqüência, é possível calcular as relações entre os elementos traços/potássio no magma residual em função da porcentagem de cristalização da leucita a partir de um magma sanidínico (Fig. 4).

Da mesma forma, é possível estabelecer as relações entre elementos traço nesse mesmo magma residual, em função do grau de cristalização (Fig. 5).

DISCUSSÃO DOS RESULTADOS Os coeficientes experimentais de partição de $\mathrm{Rb}, \mathrm{Ca}, \mathrm{Ba}$ e $\mathrm{Sr}$, entre leucita e solução hidrotermal e entre magma sanidínico e solução hidrotermal equivalente, a $1030^{\circ} \mathrm{C}$ e $2 \mathrm{kbar}$ (Tab. 1), segundo Moreira et al. (1989a, b,c,d), permitiram a obtenção indireta dos coeficientes de partição desses elementos entre a leucita e o magma sílico- sanidínico em equflibrio, a $2 \mathrm{kbar}$, num intervalo de temperatura entre aproximadamente $1.080^{\circ} \mathrm{C}$ e $980^{\circ} \mathrm{C}$ (Tab. 2) - esta última, temperatura do ponto peritético a 2 kbar (Shaw 1963).

Esses coeficientes demonstram a preferência do $\mathrm{Rb}$ e do $\mathrm{Ba}$ pela leucita em detrimento da fusão sílico-sanidínica. $\mathrm{O}$ contrário acontece com o $\mathrm{Ca}$ e o $\mathrm{Sr}$, com preferência pelo magma em detrimento da leucita.

A figura 2 mostra que com a cristalização de $30 \%$ de

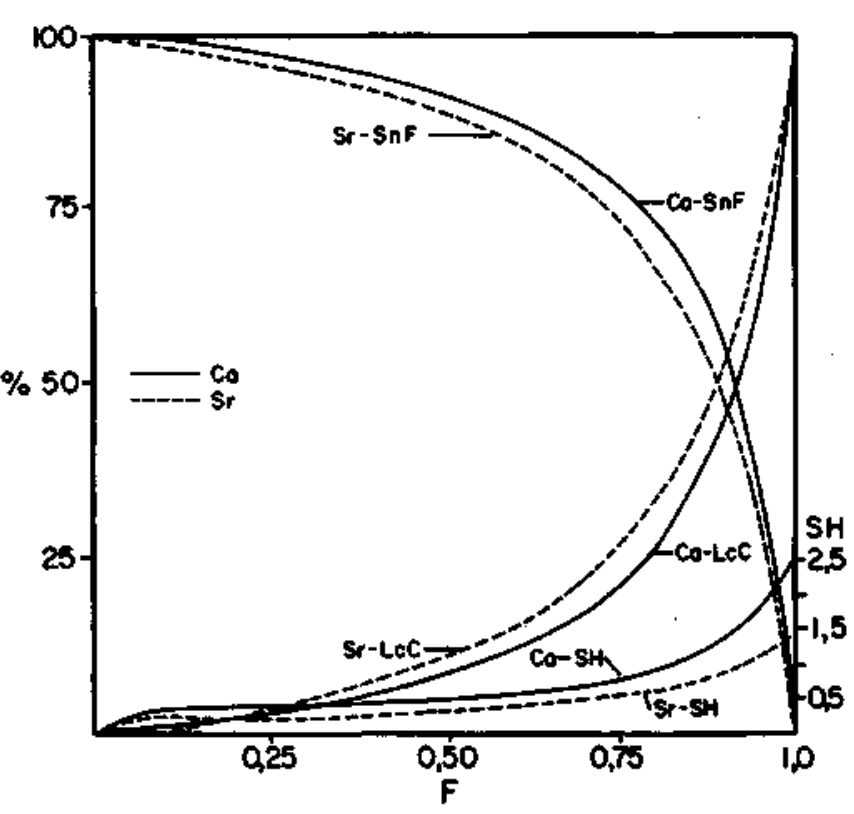

Figura 3 - Evolução da distribuição de Ca e Sr entre leucita (LcC), fusão sttico-sanidínica (SnF) e solução hidrotermal (SH) em Junção do grau de cristalização da leucita (F). Como na figura 2, os teores iniciais de Ca e Sr no "magma" experimental primitivo são tidos como $100 \%$.

Figure 3 - Evolution of $\mathrm{Ca}$ and $\mathrm{Sr}$ distributed among leucite (LcQ, silic-sanidinic fusion $(\mathrm{SnF})$ and hydrothermal solution $(\mathrm{SH})$ as function of degree of crystallization of leucite (F). As figure 2, the initial concentration of $\mathrm{Ca}$ and $\mathrm{Sr}$ in original magma are equal to $100 \%$

leucita, $50 \%$ de todo o $\mathrm{Rb}$ contido no magma inicial estaria fazendo parte da fase cristalizada. Com $50 \%$ de cristalização; aproximadamente $70 \%$ do $\mathrm{Rb}$ inicial estaria contido nas leucitas. Esses números são pouco menores para o $\mathrm{Ba}$.

A figura 3 mostra que se $30 \%$ da leucita fosse cristalizada, aproximadamente $95 \%$ do $\mathrm{Ca}$ e $\mathrm{Sr}$ iniciais estariam distribuídos na fase fundida. Quando a cristalização atingisse $50 \%$, a massa dos cristais de leucita conteria teores aproximados de $10 \%$ do Ca e Sr iniciais e os restantes $90 \%$ estariam na fase líquida.

Como conseqüência da cristalização da leucita, as relações $\mathrm{Ca} / \mathrm{K}$ e $\mathrm{Sr} / \mathrm{K}$ aumentariam exponencialmente no magma residual, em relação às mesmas relações do magma inicial. Ao mesmo tempo, as relações $\mathrm{Ba} / \mathrm{K}$ e $\mathrm{Rb} / \mathrm{K}$ diminuiriam ligeiramente no magma residual (Fig. 4).

É preciso notar que esses cálculos são normalizados à relação inicial traço/K igual a um no magma primitivo.

A figura 5 mostra os cálculos normalizados da evolução das relações entre elementos traço $\mathrm{Rb} / \mathrm{Ba}, \mathrm{Ba} / \mathrm{Sr}, \mathrm{Sr} / \mathrm{Ca}$, $\mathrm{Ba} / \mathrm{Ca}, \mathrm{Rb} / \mathrm{Ca}$ e $\mathrm{Rb} / \mathrm{Sr}$ no magma sílico-sanidínico experimental com o avanço da cristalização da leucita.

Esses dados, conseqüência direta dos trabalhos experimentais, são válidos para $2 \mathrm{kbar}$, temperaturas entre $980 \mathrm{e}$ $1080^{\circ} \mathrm{C}$, dentro dos teores de elementos traços em que os valores de Kd (determinados experimentalmente) são constantes. Outra condição de validade é que as trocas K-elemento traço ocorram em equílibrio a cada tempo, atingindo a totalidade dos cristais previamente cristalizados (até o núcleo) independente de suas dimensões e independente de considerações de ordem-desordem da leucita.

$\mathrm{O}$ modelo aqui estabelecido pode ser usado para processos de diferenciação por cristalização fracionada e segregação magmática. Basta para isso que cada magma derivado pela segregação de leucitas seja considerado para novas diferencia- 


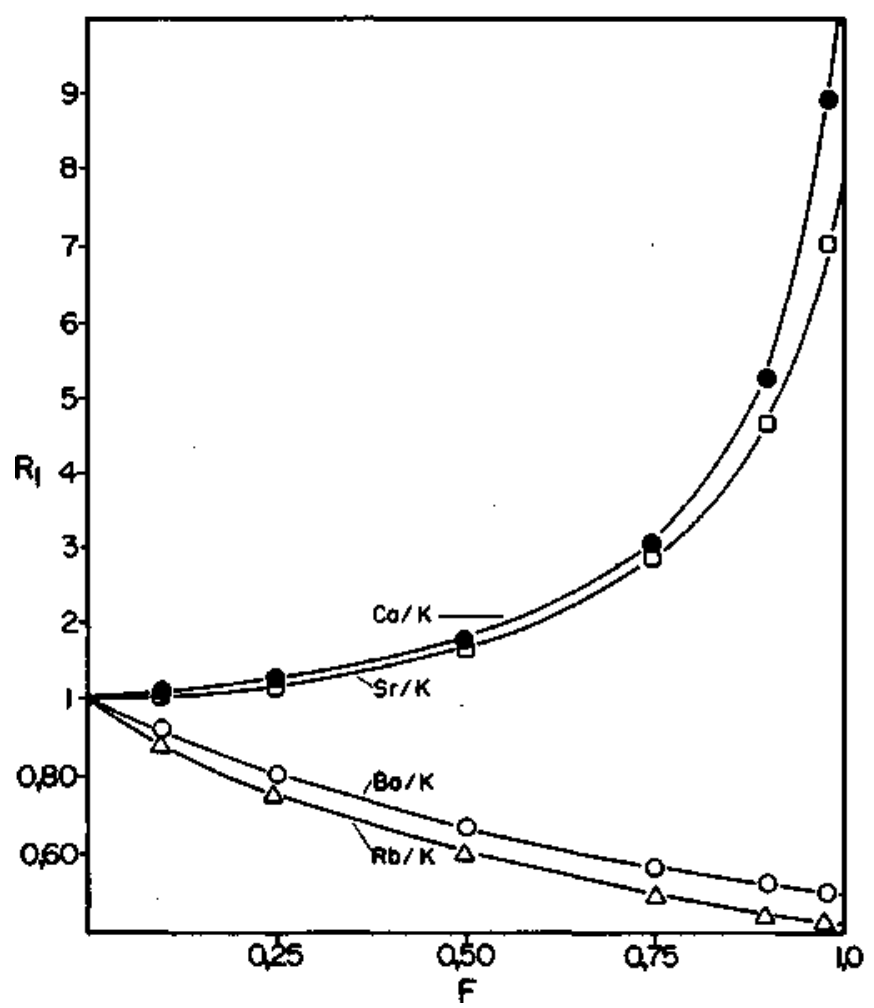

Figura 4-Evolução das relações entre os elementos traços e potássio na fusão sílico-sanidínica residual experimental, em função da cristalização da leucita $(F)$. Essas relações são tomadas como unitárias no "magma" original. A escala vertical para valores maiores que 1 é diferente dos valores menores que a unidade. $R,=\left(E T_{1} / K\right)_{\text {magma residual }}\left(E T_{1} / K\right)_{\text {magma primitivo; }} E T$ = elemento traço

Figure 4 - Evolution of trace elements and potassium relations for silicsanidinic fusion, as function of crystallization of leucite (F). These relations are equal one for original magma. The vertical scale is different for values greater than one for that lesser than one. $R_{1}=\left(E_{1} / K\right)_{\text {residual }}$ magma $/(\mathrm{ET} 1 / \mathrm{K})_{\text {original magma }}{ }^{\prime} \mathrm{ET}=$ trace element

ções, ainda acima do ponto peritético, com ajuda dos cálculos normalizados (Moreira 1988).

Em condições normais de cristalização, abaixo da temperatura peritética, a leucita reagirá com o líquido residual formando feldspato potássico (sanidina) a altas temperaturas, segundo a reação genérica:

$$
\left(\mathrm{KA} \mathrm{Si}_{2} \mathrm{O}_{6}\right)_{\mathrm{C}}+\left(\mathrm{SiO}_{2}\right)_{\mathrm{F}}=\left(\mathrm{KAlSi}_{3} \mathrm{O}_{8}\right)_{\mathrm{c}}
$$

Nesse caso, para o controle da partição dos elementos traços contidos na leucita, na fusão e no feldspato seria necessário o estabelecimento dos valores dos coeficientes de distri-

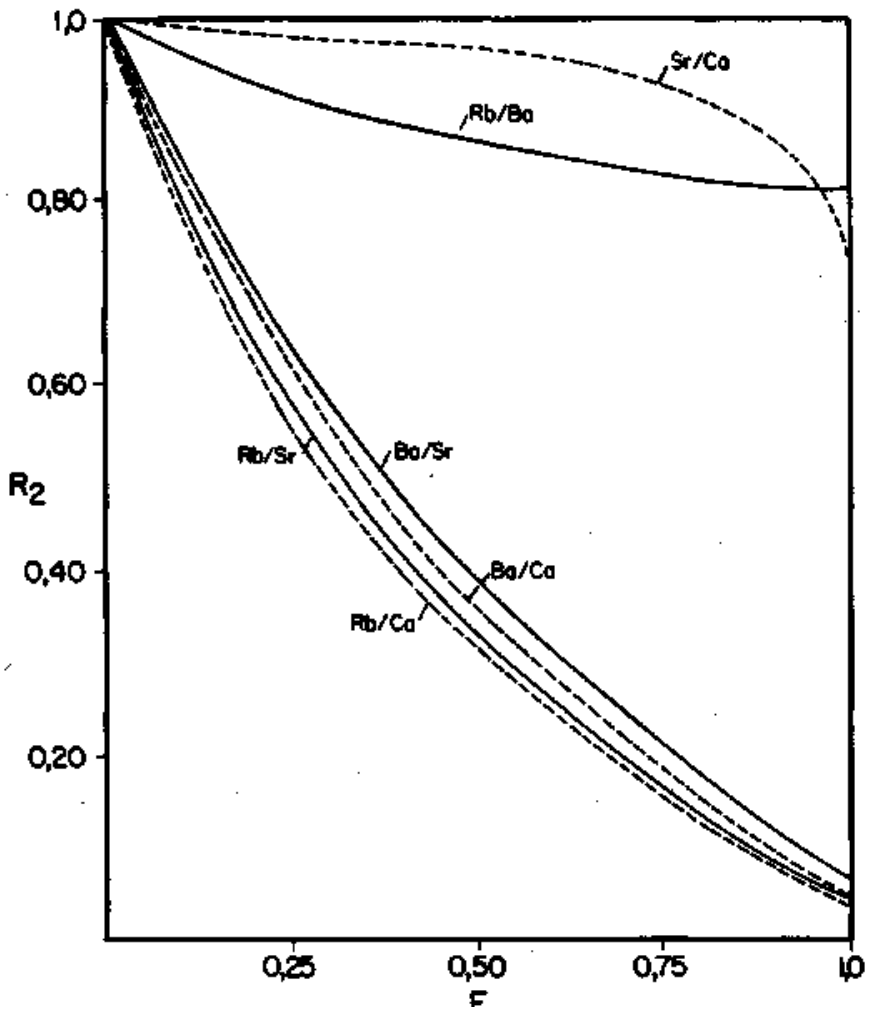

Figura 5-Evolução das relações entre os elementos traços na fusão sílico-sanidínica residual experimental em função do grau de cristalização $(F)$ da leucita. Essas relações no "Magma" inicial (vide texto) são tidas como unitárias. $R_{2}=\left(\mathrm{Et}_{1} / \mathrm{ET}_{2}\right)_{\mathrm{magma}}$ residual $\left(E T_{1} / E T_{2}\right)$ magma prlmitlvo; $E T=$ elemento traço

Figure 5 - Evolution of trace elements in silic-sanidinic fusion as function of degree of crystallization of leucite $(\mathrm{F})$. Those relations are assigned to one for original magma. $\mathrm{R}_{2}=\left(\mathrm{ET}_{1} / \mathrm{ET}_{2}\right)_{\text {residual }}$ mag. $\mathrm{ma} /\left(\mathrm{ET}_{1} / \mathrm{ET}_{2}\right)_{\text {original magma }} ; \mathrm{ET}=$ trace element

buição desses elementos entre leucita e sanidina e entre sanidina e fusão (Moreira 1988).

Agradecimentos Os autores agradecem os auxílios recebidos do Conselho Nacional de Desenvolvimento Científico e Tecnológico $(\mathrm{CNPq})$, Fundação de Amparo à Pesquisa do Estado de São Paulo (FAPESP) e (CAPES); e apoio do Centre de Recherches sur Ia Synthèse et Chimie de Minéraux (CNRS) de Orleans, do Instituto de Geociências da Universidade Federal Fluminense (IGc/UFF) e do Departamento de Mineralogia e Petrologia/Instituto de Geociências da Universidade de São Paulo (DMP/IG/USP).

\section{REFERÊNCIAS BIBLIOGRÁFICAS}

DELBOVE, F. 1978. Étude expénmentale de la distribution des alcalins $\mathrm{K}, \mathrm{Rb}$, Cs et des alcalino-terreux $\mathrm{Ca}, \mathrm{Sr}, \mathrm{Ba}$ entre albite cristállisée et fondue et solution hydrothermale. Bull Mineral., 101:317-333.

IIYAMA, J.T. 1972. Fixation des elements alcalino-terreux Ba, Sr et Ca dans lês feldspaths - Etude expénmentale. In: INT. GEOL. CONGR., 24, Montreal, 1972. Proceeding... Montreal, IUGS p.122-130.

LAGACHE, M. 1974a. Calcul des propriétés thermodynamiques des solutions ternaires de leucite par lê modele de Kohler. Application expénmentale dans lê cas ou l'un des composants est à l'êtat de traces. C.R. Acad .Sci.faris, 278:2497-2500.

LAGACHE, M. 1974b. Utilizations du modèle de F.Kohler pour le calcul de la distribution d'un élément en trace entre une solution solide binaire et une solution hydrothermale. Bull. Soc. Franc. Mineral. Cristall., 97:117-121.

MOREIRA, A.H.P. 1988. Sintese de leucita, sanidina e vidro sanidinico a 930-1030 ${ }^{\circ} \mathrm{C}$ e $2 \mathrm{~kb}$ : Coeficientes de partição de $\mathrm{Rb}, \mathrm{Ca}$, Sr e Ba entre essas fases e solução hidrotermal de KCl. Aplicações geológicas. São Paulo. 149p. (Tese de Doutoramento, Instituto de Geociências, USP).

MOREIRA. A.H.P.; DELBOVE. F.; VALARELLI. J.V. 1989a Partição de Ba entre fluidos hidrotermais $\left(930-1030^{\circ} \mathrm{C}\right.$ e $\left.2 \mathrm{kbar}\right) \mathrm{e}$ leucita, sanidina ou fusão sílico-sanidinfca. Rev. Bras. Geoc., 19(4):436-439.

MOREIRA, A.H.P.; DELBOVE, F.; VALARELLI, J.V. 1989 b. 
Partição experimental do ST entre fluido hidrotermal $\left(\mathrm{H}_{2} \mathrm{O}-\mathrm{KCl}-\mathrm{SrCl}_{2}\right)$ e leucita, sanidina e fusão sílico-sanidínica a 2 $\mathrm{kb}$ e altas temperatas \& Anais da Acad bras Ciênc.61(3):275-284.

MOREIRA, A.H.P.; DELBOVE, F.; VALARELLI, J.V. 1989c. Coeficiente de distribuição do Rb entre solução hidrotermal, sanidina, leucita e fusão sílica-sanidínica a altas temperaturas e 2 kb. Geochinica Brasiliensis. (No prelo).

MOREIRA A.H.P.; DELBOVE, F.; VALARELLI, J.V.1989d.

Coeficiente de partição do Ca entre leucita, sanidina, fusão ślico-sanidínica e solução hidrotermal a $2 \mathrm{~kb}$ e altas temperatura $\left(930^{\circ} \mathrm{Ce} 1030^{\circ} \mathrm{O}\right.$.Boletím IG-USP, 21:1-12.(Série Científica).

SHAW, H.R. 1963. The four-phase curve sanidine-quartz-liquid-gas between SOO and 4.000 bars.Amer. Miner. 18:883-896.
VOLFINGER, M. \& ROBERT, J.L. 1980. Structural control of the distribution of trace elements between silicates and hydrothermaT solutions. Geoch. Cosmoch, Acta, 44:1455-1461.

WYART, J. \& SABATIER, G. 1965. Réctions des feldspaths alcalins avec des solutions hydrothermales de $\mathrm{CaCl}_{2}$. C JR. Acad. Sá. Paris. 260:1681-1685.

MANUSCRITO A625

Recebido em 27 de outubro de 1989

Revisão do autor em 13 de fevereiro de 1990

Revisão aceita em 14 de fevereiro de 1990 